\title{
DRUG THERAPY OF AUTISM SPECTRUM DISORDERS
}

\author{
Svetlana Georgieva ${ }^{1}$, Yana Koleva ${ }^{2}$ \\ ${ }^{1}$ Department of Pharmaceutical Sciences, Faculty of Pharmacy, \\ Medical University of Varna and ${ }^{2}$ Department of Organic Chemistry, \\ Faculty of Natural Sciences, Prof. Assen Zlatarov University of Bourgas
}

\begin{abstract}
What role do medications play in the overall treatment of autistic spectrum disorders (ASD)? There have been few controlled trials of medications for this pathology. The identification of such medications awaits a more sophisticated elucidation of the genetic and neurobiological underpinnings of ASD. The aim of this paper is to present a literature review on the variety of drugs used in ASD treatment as well as on the pharmacological effects and advances in the complex treatment of patients with autism.
\end{abstract}

Key words: autism, autism spectrum disorders, interventions, drugs, medications

\section{INTRODUCTION}

Autism is a life-long developmental disability which can affect communication, social interaction, and behaviour. Its clinical form and severity can vary from person to person, however, people with autism share some difficulty in making sense of the world. First illustrated in a series of case histories by Leo Kanner in 1943, it was not until 1980 that autism was officially recognized as a disorder of development distinct from childhood schizophrenia (18).

Subjects with features similar to classic, Kannertype autism have been identified who do not fulfil the same specific criteria for autism. To recognise this, a broader range of pervasive developmental disorders including Asperger's syndrome, pervasive developmental disorder - not otherwise specified (PDD NOS)/atypical autism are now considered to be part of the autistic spectrum (although classification

Address for correspondence:

Svetlana Georgieva, PhD

Department of Pharmaceutical Sciences,

Faculty of Pharmacy, Medical University of Varna

55 Marin Drinov Str., 9002 Varna, Bulgaria

e-mail:fotkova@abv.bg

Received: February 14, 2013

Accepted: March 22, 2013 is contentious). This is as a whole known as autism spectrum disorder (ASD).

Core features of ASD are evident in three of the following behavioural areas (7):

1. Social interaction - general difficulty with and lack of interest in developing social relationships. Not understanding fully the meaning of gestures, facial expressions, body postures, voice tone, and social cues to convey meaning and regulate social interactions. An absence of insight into what the other person is thinking (lacking in a 'theory of mind') or feeling (empathy).

2. Language and communication - language deficits lead to a delay or limitations in speech, including muteness in some people and difficulty in initiating or sustaining a conversation with others. Stereotyped and repetitive use of language which can be very literal, idiosyncratic and overly formal, marked by 'over-learned scripts'. Speech is used to communicate needs and provide information rather than to exchange information or for social conversation.

3. Restrictive interests and repetitive behaviour - rigid and repetitive behaviour patterns including non-functional routines or rituals 
(e.g., touching doorknobs or walls). Inflexible adherence to behaviour and resistance to change in routine or to being interrupted. Stereotyped and repetitive motor mannerisms such as 'hand flapping' when aroused or behaviours which are pleasurable such as spinning car wheels or watching the same video repeatedly, and oral stereotyped movements such as humming or incessant questioning.

Associated ASD features sometimes evident include the following (2):

* unprovoked aggressive or violent behaviour to self and others such as biting, head-banging and gouging;

* attention and concentration problems;

* sleep disturbance and a decreased need for sleep;

* unusual responses to sensory stimuli, and

* special skills and interests such as a talent for music, mathematics, visual-spatial abilities, or an exceptional memory for areas of knowledge of particular interest. Some individuals may have an outstanding rote visual or auditory memory and a high IQ.

The triad of core features described above is the basis for the diagnostic criteria used by the Diagnostic and Statistical Manual of Mental Disorders (DSMIV) (1) and the International Classification of Diseases (ICD-10) (19) to classify ASD (Table 1).

Table 1. Diagnoses relevant to ASDs

\begin{tabular}{ll}
\hline Classification System & Disorders/syndromes \\
DSM-IV & Autistic disorder \\
& PDD NOS \\
& Asperger's syndrome \\
& Rett's disorder \\
& Childhood disintegrative \\
& disorder \\
& Childhood autism \\
& Atypical autism \\
Asperger's syndrome & Rett's syndrome \\
& Other PDDs \\
& PDD - unspecified \\
\hline
\end{tabular}

The aim of this study is to present a literature review about the treatment of humans with autism and to identify the most effective drugs of best practice in ASD treatment.

The goal in using medications is to reduce behaviours, thoughts, or emotions that interfere with education or social interaction and distress of child or others.

As a result, medications may improve the effect of non-medication therapies.

New medications are being developed all the time offering new hope for medical treatment of autism.

\section{PHARMACOLOGICAL INTERVENTIONS}

A variety of drugs are administered to treat or manage symptoms and associated conditions of autism.

\section{Typical antipsychotics}

Several studies are devoted too various typical antipsychotics including haloperidol (5) and pimozide (12). While both are considered to be efficacious, concerns about the development of dyskinesias including tardive dyskinesia (TD) have meant that usage of typical antipsychotics, particularly haloperidol, has usually been reserved for individuals with severe symptoms. One of the benefits of haloperidol is that weight change is a less common side effect of treatment.

\section{Atypical antipsychotics}

Atypical antipsychotics are recognized for their ability to produce an antipsychotic effect in a majority of patients without inducing significant extrapyramidal side effects. These effects include both early-onset and chronic neurological adverse events involving voluntary and involuntary musculature, the most common of which include parkinsonism (bradykinesia, rigidity, tremor), dystonias, and akathisia. Neuroleptic malignant syndrome is another major acute side effect that may be associated with the use of antipsychoticm drugs such as neuroleptics. It is typically characterised by severe muscular rigidity, fever, an altered level of consciousness, and autonomic instability. Atypical antipsychotics, i.e. potent antagonists of serotonin and dopamine increased in popularity, given the 
lower TD incidence rate in the short term, and clinicians began to use them more often when treating the individuals with autism.

Risperidone is effective in improving the hyperactivity and reducing the frequency and intensity of temper outbursts and aggression, which are sometimes apparent as associated symptoms of people with autism, although there are concerns about its side effects such as sedation and weight gain (11). At higher doses, extrapyramidal side effects may occur more frequently.

Olanzapine has been used for treatment of aggression, hyperactivity, mood symptoms, sleep disturbances and psychosis in patients with autism (14). Weight gain may be a problematic side effect with this medication, too.

Clozapine usage has been described in a small case study of only three children (20). Its limited use to date may relate to the need for frequent tests to monitor leukocyte counts due to the risk of agranulocytosis as well as to a higher risk of seizures at high doses. This is particularly noteworthy as individuals with autism are already at higher than normal risk of developing seizure disorders.

\section{Antidepressants}

A wide variety of antidepressant medications are used in the treatment of autism for decades. This drug class comprises a broad group of chemical compounds that generally work by increasing the concentration in the synaptic space primarily of noradrenaline and/or serotonin (5-HT). Researchers investigate whether the selective serotonin re-uptake inhibitors (SSRIs) may be of benefit in reducing the repetitive behaviours, aggression and aspects of social relatedness in children, adolescents, and adults with autism. These medications are considered safer alternatives than the tricyclic antidepressants, given their better tolerability and lack of significant cardiac effects.

Clomipramine is a tricyclic antidepressant recognized for its potent inhibition of 5-HT uptake, i.e., acting as a non-selective SRI. It is more efficacious than the relatively selective noradrenaline-reuptakeinhibiting tricyclic desipramine in the treatment of children with obsessive-compulsive disorder (10). The repetitive behaviours and stereotyped movements commonly seen in PDD including ASD resemble, in part, the compulsions observed in this disorder. Clomipramine efficacy is studied in children, adolescents and adults with autism. There is some suggestion that this drug could be better tolerated by adolescents and adults than by children.

As potent inhibitors of 5-HT uptake, SSRIs such as fluvoxamine have received increasing attention as a treatment option for autistic symptoms because of their better side effect profiles compared to tricyclics such as clomipramine. Fluvoxamine appears to target a range of symptoms including repetitive thoughts and behaviour, maladaptive behaviour, aggression, and communicative use of language. However, its lower efficacy and tolerability has been established in children and adolescents with autism (17).

Other examples of antidepressant medication such as imipramine, trazodone, bupropion, venlafaxine, nefazadone, monoamine-oxidase inhibitors (MAOIs) and mirtazapine may be used for managing ASD symptoms.

\section{Fenfluramine}

Fenfluramine is an indirect 5-HT receptor agonist with structural similarities to amphetamine. Several initial reports indicate that this agent is promising as a potential treatment for autism. An initial double-blind crossover study of fenfluramine and placebo displays favourable results. However, subsequent double-blind placebo-controlled trials failed to show consistent benefits with fenfluramine use in children. Approved by the Food and Drug Administration (FDA) for the treatment of obesity, the drug is voluntarily withdrawn from the world market in 1997 because of concern that it contribute to the development of cardiac valvular disease (13).

\section{Stimulants}

Early studies of the effects of stimulants in children with autism generally produce negative results. There are concerns about the possibility that stimulants may lower the seizure threshold. This leads to the widespread clinical view that stimulants are contraindicated in treating autism (13). However, some preliminary studies with children suggest positive benefits with stimulant treatment relating to motor hyperactivity and inattention in autism.

\section{Anticonvulsants}

It remains unclear whether anticonvulsants such as lamotrigine may be helpful in improving other clinical symptoms of autism in adolescents 
and adults, in addition to their effects in controlling seizure disorders (3) although some preliminary work with sodium valproate is promising.

\section{Lithium}

Lithium is an alkaline metal salt that acts on multiple neurotransmitter systems. This agent could be effective for some individuals with ASD where there are symptoms resembling mania and positive family histories of bipolar disorder (9).

\section{Anxiolytics}

Buspirone is a 5-HT1A receptor partial agonist used in the treatment of a generalized anxiety disorder that may improve target symptoms of anxiety and irritability in the patients with PDD including autism, too (4).

\section{a2-Adrenergic agonists}

Guanfacine is applied to improve hyperactivity, inattention, insomnia and tics in individuals with autism (17).

\section{$\beta$-Adrenergic antagonists}

Propanolol is used in treating aggression and self-injury in adults with autism and exerts a positive effect on socialization and speech (15).

\section{Opiate antagonists}

Excess opiate activity is mooted as a contributing factor to the aberrant social and language development observed in individuals with autism. The opiate antagonist naltrexone hydrochloride is investigated for its efficacy in treating the core symptoms of autism and reducing the self-injurious behaviour (17).

\section{Miscellaneous}

Secretin, a gastrointestinal polypeptide hormone, is an agent recently received attention as a possible pharmacological treatment for ASD. Porcine secretin as well as synthesized human secretin administered intravenously is applied. Originally FDA-approved for use in the diagnosis of certain gastrointestinal diseases, secretin's use for the treatment of autism is popularized by a small study that reports its positive impact on language and social relatedness in three small children with autism (8).

Vancomycin and intravenous immunoglobulin are investigated to reveal if their usage results in any behavioural improvements in children with autism (16).
There are claims that 'mega' dosages of certain vitamins and other agents could ameliorate or prevent a range of pathoilogical conditions including ASD. Such agents include vitamin B6, vitamin B12, folic acid, magnesium and melatonin. In general, vitamins act as coenzymes or enhancers of biochemical processes, many of which are involved in the synthesis and regulation of neurotransmitters and other polypeptide products. High doses of vitamins, especially pyridoxine or vitamin B6 in conjunction with magnesium are proposed as being helpful in autism by reducing the aggressiveness, self-stimulation, and avoidance of eye contact, and improving the social relatedness and speech. Mega dosages of vitamins are variable in their toxicity, depending on the vitamin used. So, the effectiveness of vitamin therapy is questioned (6).

\section{DIRECTIONS FOR FUTURE RESEARCH}

Future research should attempt to address the methodological limitations in study of ASD and they are expected to lead to better quality of life of the patients with ASD.

Some features of future research are briefly presented below:

* Consideration of a broader range of pharmacological interventions and trials comparing clinically acceptable drugs;

* Studies comparing pharmacological interventions within and between drug classes, including polypharmacy;

* Studies systematically comparing multiple therapies (pharmacological, behavioral, and educational) or combinations of these;

* Studies with longer follow-up periods for assessing outcomes post-treatment, particularly safety outcomes, given that autism is a chronic condition and long periods of treatment are to be expected;

- Studies with multivariate analyses to investigate the effects of potentially confounding factors in intervention effectiveness;

* More generally, studies are required of the differences between subtypes of autism across ASD as well as of the varying treatment and 
Svetlana Georgieva, Yana Koleva

support needs of these distinct diagnostic groups;

* With respect to multiple pharmacotherapy interventions, drugs that may treat one symptom may exacerbate another, requiring the development of more selective agents, and

* Molecular genetic studies of extensive interest may lead to the development of new and effective treatments.

\section{CONCLUSION}

Whilst autism currently cannot be cured, pharmacological interventions are utilized to assist in managing the symptoms and associated conditions of autism. These may be problematic for the person with autism and/or their carers. Such treatments may also enhance the benefit from other concurrent therapies which include behaviour modification and other psychosocial and educational interventions. Medications are rarely the sole treatment modality for any individuals with autism. As an adjunctive treatment they may be effective with relatively few and manageable side effects, although to date evidence is very limited. Many medications that appear promising in small, open-label studies are not studied under reliable experimental conditions in any age group yet.

While more research is required of agents that target associated symptoms interfering with functioning or causing distress, development and evaluation of promising medications concerning the treatment of core symptoms of social and language impairment in ASD should be a priority, too.

\section{REFERENCES}

1. American Psychiatric Association. Diagnostic and statistical manual of mental disorders: DSMIV. Washington, DC: American Psychiatric Association, 1994.

2. Aylott, J. Autism in adulthood: the concepts of identity and difference.- Br. J. Nurs., 9, 2000, No 13, 851-858.

3. Belsito, K. M., P. A. Law, K. S. Kirk,R. J. Landa, A. $\mathrm{W}$. Zimmerman. Lamotrigine therapy for autistic disorder: a randomized, double-blind, placebocontrolled trial.- J. Autism Dev. Disord., 31, 2001, No 2, 175-181.
4. Buitelaar, J. K., R. J. van der Gaag, J. van der Hoeven. Buspirone in the management of anxiety and irritability in children with pervasive developmental disorders: results of an open-label study.- J. Clin. Psychiatry, 59, 1998, No 2, 56-59.

5. Campbell, M., L. T. Anderson, M. Meier, I. L. Cohen, A. M. Small, C. Samit, et al. A comparison of haloperidol and behavior therapy and their interaction in autistic children.- J. Am. Acad. Child Psychiatry, 17, 1978, No 4, 640-655.

6. Findling, R. L., K. Maxwell, L. Scotese-Wojtila, J. Huang, T. Yamashita, M. Wiznitzer. Highdose pyridoxine and magnesium administration in children with autistic disorder: an absence of salutary effects in a double-blind, placebocontrolled study.- J. Autism Dev. Disord., 27, 1997, No 4, 467-478.

7. Folstein, S. E., B. Rosen-Sheidley. Genetics of autism: complex aetiology for a heterogeneous disorder.- Nat. Rev. Genet., 2, 2001, No 12, 943-955.

8. Horvath, K., G. Stefanatos, K. N. Sokolski, R. Wachtel, L. Nabors, J. T. Tildon. Improved social and language skills after secretin administration in patients with autistic spectrum disorders.- J. Assoc. Acad. Minor. Phys., 9, 1998, No 1, 9-15.

9. Kerbeshian, J., L. Burd, W. Fisher. Lithium carbonate in the treatment of two patients with infantile autism and atypical bipolar symptomatology.- J. Clin. Psychopharmacol., 7, 1987, No 6, 401-405.

10. Leonard, H. L., S. E. Swedo, J. L. Rapoport, E. V. Koby, M. C. Lenane, D. L. Cheslow, et al. Treatment of obsessive-compulsive disorder with clomipramine and desipramine in children and adolescents: a double-blind crossover comparison.Arch. Gen. Psychiatry, 46, 1989, No 12, 1088-1092.

11. McDougle, C. J., J. P. Holmes, D. C. Carlson, G. H. Pelton, D. J. Cohen, L. H. Price. A doubleblind, placebo-controlled study of risperidone in adults with autistic disorder and other pervasive developmental disorders.- Arch. Gen. Psychiatry, 55, 1998, No 7, 633-641.

12. Naruse, H., M. Nagahata, Y. Nakane, K. Shirahashi, M. Takesada, K. Yamazaki. A multi-center doubleblind trial of pimozide (Orap), haloperidol and placebo in children with behavioral disorders, using crossover design.- Acta Paedopsychiatr., 48, 1982, No 4, 173-184. 
13. Posey, D. J., C. J. McDougle. The pharmacotherapy of target symptoms associated with autistic disorder and other pervasive developmental disorders.- Harv. Rev. Psychiatry, 8, 2000, No 2, 45-63.

14. Potenza, M. N., J. P. Holmes, S. J. Kanes, C. J. McDougle. Olanzapine treatment of children, adolescents, and treatment adults with pervasive developmental disorders: an open-label pilot study.J. Clin. Psychopharmacol., 19, 1999, No 1, 37-44.

15. Ratey, J. J., Mikkelsen, E., Sorgi, P., Zuckerman, H. S., Polakoff, S., Bemporad, J., et al. Autism: the treatment of aggressive behaviors.- J. Clin. Psychopharmacol., 7, 1987, No 1, 35-41.

16. Sandler, R. H., S. M. Finegold, E. R. Bolte, C. P. Buchanan, A. P. Maxwell, M. L. Väisänen, et al. Short-term benefit from oral vancomycin treatment of regressive-onset autism.- J. Child Neurol., 15, 2000, No 7, 429-435.
17. Stigler, K. A., D. J. Posey, C. J. McDougle. Recent advances in the pharmacotherapy of autism.Expert Rev. Neurother., 2, 2002, No 4, 499-510.

18. Wing, L. The autistic spectrum: a guide for parents and professionals. London, Constable, 1996.

19. World Health Organization. The ICD-10 classification of mental and behavioural disorders: clinical descriptions and diagnostic guidelines. Geneva, World Health Organization, 1992.

20. Zuddas, A., M. G. Ledda, A. Fratta, P. Muglia, C. Cianchetti. Clinical effects of clozapine on autistic disorder.- Am. J. Psychiatry, 153, 1996, No 5, p. 738. 